\title{
Implementing good participatory practice guidelines in the FEM-PrEP Preexposure Prophylaxis Trial for HIV Prevention among African Women: a focus on local stakeholder involvement
}

\author{
This article was published in the following Dove Press journal: \\ Open Access Journal of Clinical Trials \\ 24 October 2013 \\ Number of times this article has been viewed
}

Natasha Mack'
Stella Kirkendale'
Paul Omullo ${ }^{2}$
Jacob Odhiambo ${ }^{2}$
Malebo Ratlhagana ${ }^{3}$
Martha Masaki ${ }^{4}$
Phumzile Siguntu'
Kawango Agot
Khatija Ahmed
Saidi Kapiga
Johan Lombaard
Lut Van Damme'
Amy Corneli'
'FHI 360, Durham, NC, USA;
${ }^{2}$ Impact Research and Development
Organization, Bondo, Kenya; ${ }^{5}$ Setshaba
Research Centre, Soshanguve,
South Africa; ${ }^{4}$ Kilimanjaro Christian
Medical Center, Moshi, Tanzania; ${ }^{5}$ Josha
Research, Bloemfontein, South Africa

Correspondence: Natasha Mack FHI 360, 2224 E North Carolina Highway 54, Durham, NC 277I3, USA $\mathrm{Tel}+$ I 9 | 95447040 ext | |47|

Fax + I919544726I

Email nmack@fhi360.org

\begin{abstract}
Biomedical HIV-prevention research is most likely to succeed when researchers actively engage with community stakeholders. To this effect, the Joint United Nations Programme on HIV/AIDS and the AIDS Vaccine Advocacy Coalition developed good participatory practice guidelines for biomedical HIV-prevention trials in 2007 and updated them in 2011. The Preexposure Prophylaxis Trial for HIV Prevention among African Women (FEM-PrEP) clinical trial, testing once-daily Truvada as preexposure prophylaxis among women at higher risk of HIV in Kenya, South Africa, and Tanzania, included a community program to engage with local stakeholders. Following the trial, we revisited the community program to situate activities in the context of the 2011 guidelines. In the paper, we describe implementation of the six guidelines relevant to local stakeholder engagement - stakeholder advisory mechanisms, stakeholder engagement plan, stakeholder education plan, communications plan, issues management plan, trial closure, and results dissemination - in light of on-the-ground realities of the trial. We then identify two cross-cutting themes from our considerations: (1) stakeholder education beyond the good participatory practice recommendation to increase research literacy about the specific trial is needed; education efforts should also communicate a base of information on HIV transmission and prevention; and (2) anticipatory preparation is useful in communications planning, issues management, and trial closure and results dissemination, and may contribute to successful conduct of the trial; in FEM-PrEP, this was possible through integration of the community program with social, behavioral, and clinical research.
\end{abstract}

Keywords: stakeholder engagement, community engagement, community involvement, HIV-prevention trials, good participatory practice guidelines, FEM-PrEP

\section{Introduction}

The controversies surrounding the trials of HIV preexposure prophylaxis (PrEP) in Cambodia and Cameroon in 2005 highlighted the need to expand stakeholder engagement in biomedical HIV-prevention clinical trials beyond study populations alone, to include also nongovernmental organizations (NGOs) and local, national, and international stakeholders. ${ }^{1-5}$ These controversies also underscored the need for adequate lead time for relationship-building with local and international stakeholders before a clinical trial is implemented. ${ }^{2,4}$

Along with this expanded vision of community stakeholder engagement, a paradigm has emerged in the past decade whereby local community partners and researchers look to each other for guidance throughout the research process. ${ }^{6}$ For example, researchers 
might establish and consult with the study's community advisory board (CAB) prior to initiation of a trial to learn whether particular visit procedures are acceptable to the potential study population, local community representatives might alert research staff to local concerns and suggest approaches for addressing them, and study staff may conduct research-literacy training for $\mathrm{CABs}$ or other local community partners to ensure that they are able to ask critical questions and serve as an effective liaison between researchers and the local community. This interactive approach to local stakeholder engagement often leads to a collaborative relationship between researchers and the local community and builds research literacy capacity in the community, which could lead to a decrease in rumors and misinformation in the community and increased local understanding of clinical research and continued support of trials. ${ }^{7}$

In response to the global dialogue on community stakeholder engagement that emerged from the Cambodia and Cameroon PrEP trial controversies, the United Nations Programme on HIV/AIDS and the AIDS Vaccine Advocacy Coalition (AVAC) developed guidelines for community engagement that were first published in 2007 as Good Participatory Practice Guidelines: Biomedical HIV Prevention Trials. Fifteen good participatory practice (GPP) guidelines formed the core of the original document; the guidelines were revised in 2011 to capture emerging lessons learned in HIV-prevention trials and comprised 16 guidelines as of that date (Table 1). One of the changes to the 2011 version was the replacement of the term "community engagement" with the term "stakeholder engagement," supporting a movement away from the perceived narrow involvement of trial communities in research implementation to one of multisector partnerships between researchers, communities, local and national governments (eg, public health officials, ward counselors, and other elected officials), and civil society stakeholders (eg, NGOs, community-based

Table I The 2007 and 20 I I GPP guidelines and corresponding FEM-PrEP trial components

\begin{tabular}{|c|c|c|}
\hline 2007 GPP guidelines & 20 I I GPP guidelines & FEM-PrEP trial components \\
\hline Formative research with the community & Formative research activities & Social and behavioral research \\
\hline Community advisory mechanisms & Stakeholder advisory mechanisms & Community program \\
\hline \multirow[t]{2}{*}{ Community engagement/involvement/education plan } & Stakeholder engagement plan & Community program \\
\hline & Stakeholder education plan & Community program \\
\hline \multirow[t]{2}{*}{ Communications plan } & Communications plan & $\begin{array}{l}\text { Community program (local } \\
\text { communications) }\end{array}$ \\
\hline & & $\begin{array}{l}\text { Clinical trial (national and international } \\
\text { communications) }\end{array}$ \\
\hline \multirow[t]{2}{*}{ Monitoring and issues management plan } & Issues management plan & Community program (local) \\
\hline & & Clinical trial (national, international) \\
\hline \multirow[t]{2}{*}{ Site development } & Site selection & Clinical trial \\
\hline & & Social and behavioral research \\
\hline \multirow[t]{9}{*}{ Protocol development } & Protocol development & Social and behavioral research \\
\hline & & Community program (minor contributions) \\
\hline & Informed consent process & Social and behavioral research \\
\hline & & Clinical trial \\
\hline & & Community program (contributions) \\
\hline & Standard of HIV prevention & Clinical trial \\
\hline & Access to HIV care and treatment & Clinical trial \\
\hline & & Community program (contributions) \\
\hline & Non HIV-related care & Clinical trial \\
\hline Policy on coverage for research-related harm & Policies on trial-related harms & Clinical trial \\
\hline Study initiation & Trial accrual, follow-up, and exit & Social and behavioral research \\
\hline Study conduct & & Community program (minor contributions) \\
\hline Study closure & Trial closure and results dissemination & Community program \\
\hline Data analysis, validation, dissemination and publication & & Social and behavioral research \\
\hline \multirow[t]{2}{*}{ Future access to HIV prevention technologies } & Post-trial access to trial products & Clinical trial \\
\hline & or procedures & PrEP rollout research \\
\hline Institutional review boards, ethics committees & & Clinical trial \\
\hline \multirow[t]{2}{*}{ and other regulatory mechanisms } & & Social and behavioral research (whose \\
\hline & & protocol included the community program) \\
\hline Site maintenance between trials & & $\mathrm{N} / \mathrm{A}$ \\
\hline
\end{tabular}

Abbreviations: GPP, good participatory practice; FEM-PrEP, Preexposure Prophylaxis Trial for HIV Prevention among African Women; Pr-EP, preexposure prophylaxis; N/A, not applicable. 
organizations, faith-based organizations, advocacy groups, and women's groups) in all stages of the research process.

\section{FEM-PrEP}

The Preexposure Prophylaxis Trial for HIV Prevention among African Women (FEM-PrEP) was a phase III, randomized, double-blind, placebo-controlled effectivenessand-safety clinical trial (ClinicalTrials.gov identifier NCT00625404) conducted from 2007 to 2011 to assess the role of emtricitabine/tenofovir disoproxil fumarate (Truvada) in preventing HIV acquisition among HIV-negative women. The trial sites were Bondo, Kenya; Pretoria and Bloemfontein, South Africa; and Arusha, Tanzania. The trial participants were between the ages of 18 and 35 years and at higher risk of HIV exposure, and had volunteered to take part in the trial. In April 2011, the trial was stopped early, when an independent data-monitoring committee advised that FEM-PrEP would be unlikely to demonstrate that Truvada prevents HIV infection in the study population, even if the trial continued to its originally planned conclusion. Later analysis indicated that the trial was unable to evaluate effectiveness because of poor adherence to the study product among trial participants. ${ }^{8}$

FEM-PrEP was comprised of four broad components - the clinical component, integrated social and behavioral research, the community program, and PrEP rollout research - that collectively aligned with the 15 guidelines from the 2007 GPP guidelines document, as summarized in Table 1.

Following the conclusion of FEM-PrEP, we revisited the community program activities to situate them in the context of the revised 2011 GPP guidelines (Table 1). Our goal was to consider the six guidelines specifically related to local community stakeholder engagement in light of the on-theground realities of FEM-PrEP. We describe our reflections here related to these six guidelines: stakeholder advisory mechanisms, stakeholder engagement plan, stakeholder education plan, communications plan, issue management plan, and trial closure and results dissemination. We found all to be implementable in FEM-PrEP and identified two cross-cutting themes from our considerations.

\section{Community program activities overview}

The FEM-PrEP community program focused specifically on engaging and educating local stakeholders, including the potential study-participant population and other people in the community likely to be affected by or to have an influence on the conduct of the research. The community program was part of the social and behavioral research protocol, and was referred to as social, behavioral, and community (SBC) work.

\section{Ethics statement}

Although FHI 360's Protection of Human Subjects Committee (PHSC) and the FHI 360 research team considered the community program to be a nonresearch activity, it was approved by the PHSC as part of the aforementioned study protocol in 2007 and by all site ethics review committees prior to commencement of the work.

The community program was implemented at all four trial sites: Bondo, Kenya; Pretoria and Bloemfontein, South Africa; and Arusha/Moshi, Tanzania (which were considered one trial site due to their proximity and having one principal investigator, even though they had separate formal community advisory mechanisms). We initiated the FEM-PrEP community program activities up to 2 years in advance of the clinical trial while trial start-up activities (including social and behavioral research) were taking place, as shown in Table 2. Community program activities continued throughout the course of the clinical trial, until final results were disseminated.

There were three chronological phases of community program activities in FEM-PrEP: (1) pretrial site preparedness, (2) the ongoing clinical trial, and (3) posttrial. Three objectives guided activities across all phases:

- To introduce FEM-PrEP to and obtain feedback from a range of local community stakeholders

Table 2 FEM-PrEP clinical trial and social, behavioral, and community activities timeline

\begin{tabular}{lllllll}
\hline Site & \multicolumn{2}{l}{ Site preparedness initiated } & & $\begin{array}{l}\text { CP, SBR, CT: } \\
\text { ongoing trial } \\
\text { phase initiated }\end{array}$ & $\begin{array}{l}\text { Trial } \\
\text { closure }\end{array}$ & Local results dissemination \\
\cline { 2 - 5 } & CP & SBR & Early closure & Final results \\
\hline Bondo, Kenya & June 2007 & August 2007 & May 2009 & April 20II & April-August, 20II & February-March, 2012 \\
Pretoria, South Africa & March 2008 & January 2008 & July 2009 & & April-August, 20II & February-March, 2012 \\
Bloemfontein, South Africa & March 2010 & N/A & May 2010 & April-June, 20II & February-May, 2012 \\
Arusha/Moshi, Tanzania & August 2008 & April 2008 & November 2010 & April-September, 20II & February-April, 2012 \\
\hline
\end{tabular}

Abbreviations: FEM-PrEP, Preexposure Prophylaxis Trial for HIV Prevention among African Women; CP, community program; SBR, social and behavioral research; $\mathrm{CT}$, clinical trial; N/A, not applicable. 
- To build research literacy to promote understanding of and trust in FEM-PrEP and clinical research in general

- To promote ongoing dialogue between FEM-PrEP researchers and local community stakeholders regarding concerns, rumors, and misconceptions related to the trial.

Dedicated staff experienced in community education and mobilization were engaged to develop and implement the community program at each site with the collaboration of the community program manager at FHI 360. Numbers of staff varied from site to site, but were usually one to three. Types of staff and their specific responsibilities also varied, but always included a lead community educator responsible for identifying $\mathrm{CAB}$ members representative of the community, leading and supporting $\mathrm{CAB}$ training, building positive relationships with organizations involved in HIV and other areas, developing and facilitating outreach and community education activities about HIV-prevention research, developing and disseminating culturally and linguistically appropriate community education materials, and reporting to the site principal investigator and FHI 360 on community program activities. There were sometimes also one or more community "mobilizers" who assisted with the community program's scope of work in addition to their work on the clinical trial. The SBC project coordinator and other clinical trial staff assisted community program staff with activities as needed.

\section{GPP guidelines and the community program}

Our reflections on the FEM-PrEP community program in the context of the GPP guidelines are based on our posttrial review of study documents (eg, protocol, informed consent forms), CAB agendas and meeting notes, weekly/monthly site reports to FHI 360, trip reports, work plans, communication plans, and results-dissemination plans for each site. The site-based authors of this manuscript also provided accounts of their community program activities, as well as their reflections on the challenges of the community program and what they considered to be the most valuable community program endeavors for the clinical trial.

\section{Stakeholder advisory mechanisms}

GPP recommends utilizing both formal and informal stakeholder advisory mechanisms for the life of the trial in order to "facilitate meaningful dialogue among research teams and relevant stakeholders about planned or ongoing clinical trials." ${ }^{\text {" }}$

The FEM-PrEP community program employed formal and informal stakeholder advisory mechanisms. Formal stakeholder advisory mechanisms involved collaborations with the CABs. During the preparedness phase, the community program staff led the establishment of the CABs prior to trial initiation at all sites except Moshi, which had a previously established community advisory group (CAG). We made efforts to create balanced representation from a range of local stakeholders who might be affected by the trial, including formal and informal leaders in the local trial communities and people sharing characteristics of the study population. After establishing the $\mathrm{CABs} / \mathrm{CAG}$, the community educator, site principal investigator, and FHI 360 community program manager facilitated training with $\mathrm{CAB} / \mathrm{CAG}$ members to build their research literacy and capacity using the Research Ethics Training Curriculum for Community Representatives. ${ }^{9}$ Training on the FEM-PrEP protocol was also conducted.

Throughout implementation of the trial, the $\mathrm{CABs} / \mathrm{CAG}$ participated in sessions where study staff provided information and updates on the trial. They also communicated studyrelated concerns and issues to the study team during meetings held pretrial, over the course of the trial, and at trial closure. Community program staff also held refresher trainings for $\mathrm{CAB}$ members, and the $\mathrm{CABs} / \mathrm{CAG}$ met quarterly and up to two additional times per year on an ad hoc basis.

We learned that several factors facilitated dialogue with the $\mathrm{CABs} / \mathrm{CAG}$. The members appeared to be motivated and engaged when they helped to set the agendas for the meetings, such as suggesting topics for trainings and discussion. Sites with previous clinical trial experience had better $\mathrm{CAB}$ / $\mathrm{CAG}$ attendance and appeared best facilitated when paired with community program staff who also had experience with leading community advisory mechanisms. Participation of trial leadership at $\mathrm{CAB} / \mathrm{CAG}$ meetings to provide updates and address questions and concerns helped to facilitate dialogue with the local community representatives.

Informal stakeholder advisory mechanism activities consisted of outreach meetings and regular stakeholder forums within the larger community. During the sitepreparedness phase, community educators contacted local stakeholders - including high-level government officials, community leaders, faith-based organizations, NGOs, and community-based organizations - individually or in groups to inform them of the planned clinical trial and to learn their perspectives. Meetings and forums continued with local stakeholders throughout implementation of the clinical trial, as well as outreach activities with owners of recruitment establishments (eg, taverns) and potential participants.

We found that these activities provided a fruitful context for dialogue between researchers and the local community when the activities involved community education and an opportunity for community feedback. In addition, we found 
that these informal stakeholder advisory mechanism activities were most appropriate for addressing emergent needs identified in the local community during the trial.

For example, the Bondo community program staff held "continuing medical education" meetings at health facilities with the goal of educating providers on PrEP and providing opportunities for them to express their concerns about FEM-PrEP. Even though the trial was being conducted in the context of the district hospital, many hospital staff did not understand why the trial involved giving antiretroviral drugs to people who were HIV-negative. The community program team found that these meetings played a key role in fostering an understanding of the trial.

Similarly, some FEM-PrEP community meetings focused both proactively and responsively to issues related to male involvement. This was an important topic, because we heard anecdotally that it was difficult for some women to enroll or keep up their study visits due to limited support from male partners. Men in general also had questions about the trial. When study staff introduced the study to potential participants at the Arusha site, for example, men in general wanted to know what the discussion with the women was about and why the trial was only enrolling women. After including the men in informational meetings, men expressed support for the study and appreciation for the community education sessions, especially with respect to information about HIV/AIDS and the importance of clinical trials. These meetings created platforms for the education and involvement of male partners both directly and indirectly. When certain issues arose involving male partners, research staff would speak directly with them, if requested by the study participant; community program staff also targeted communities where women were recruited, in hopes of reaching their male partners, to address misinformation about the trial, as well as provide information on current HIV-prevention strategies.

At the Bondo site, the need to involve men was identified during site-preparedness formative research and continued to emerge as an issue throughout the trial. Some men also expressed concern about not being informed about their partner's participation. Suspicions of infidelity related to trial participation were also voiced. Because limited partner support could impede participants' decisions to attend their study visits or ability to adhere to the study product, the Bondo team built relationships with the men in the community and conducted extensive outreach focusing on men.

\section{Stakeholder engagement plan}

GPP recommends developing a stakeholder engagement plan that "describes strategies and mechanisms for building relationships and constructively engaging with a broad range of local, national, and international stakeholders." ${ }^{\circ}$

Community programs at each site shared the same overall goals and types of activities, but planning for local stakeholder engagement was specific to each site and involved the codevelopment of community engagement work plans. FHI 360 provided site staff with a guidance document and template that included specific activities, the timeline, target stakeholder groups, staff responsible, and indicators to measure fulfillment of the goals and activities. Site staff then developed a plan of activities to meet local needs. Findings from the social and behavioral site-preparedness research - which had identified community education needs, suggestions for keeping the community engaged during implementation of the clinical trial, and strategies to educate potential participants and the community about the trial's scientific agenda - informed development of the activities. The community team revisited the work plan on an annual basis, reviewing whether the goals had been met and making modifications as needed. Regular reports completed by site staff also allowed for ongoing joint evaluation of progress made in meeting the work-plan objectives.

Types of activities in the work plan included outreach efforts with individuals (eg, at bars, taverns, women's groups, and sex-worker "hot spots"), broad-based and targeted stakeholder meetings, and use of a public address system during community and national public functions and market days, and health fairs. During the results-dissemination phase, different mechanisms were used to inform different stakeholders; this was strategic and deliberate, depending on the stakeholder: by letter, phone call, or face-to-face meeting.

Sites also participated in events organized by other organizations/groups, such as South Africa's Health Calendar Days. They also conducted interviews with print media and local radio. FEM-PrEP also produced a newsletter that was distributed in print form and electronically.

Although we did not overtly define the type of engagement appropriate for each stakeholder as described in the guideline, we learned it was essential that all stakeholders be informed about PrEP, PrEP research, and FEM-PrEP, as this formed the basis of stakeholder consultation, collaboration, and empowerment. We also found that providing an overall framework - consisting of a guidance document and worksheets - with shared goals coupled with flexibility to meet the local needs led to an effective initial stakeholder engagement plan. Revising that plan annually, as well as regularly documenting and evaluating progress in reaching the planned activities, was valuable in attaining the program goals. 


\section{Stakeholder education plan}

GPP recommends the development and implementation of research-literacy efforts designed to increase understanding of both a specific trial and biomedical HIV-prevention research in general. ${ }^{6}$

Stakeholder education was a substantial component of the FEM-PrEP community program. Staff sought to educate local stakeholders about FEM-PrEP and biomedical HIV-prevention research through research-literacy efforts among the CABs/CAG and other community members, including using dynamic documents they codeveloped and updated with researchers for this purpose. For the CABs/ $\mathrm{CAG}$, activities to increase research literacy consisted of formal trainings and ongoing educational updates at their regular meetings. Training in the basics of clinical trials and in FHI 360's Research Ethics for Community Representatives proved critical to $\mathrm{CAB}$ members' understanding of such concepts as randomization, placebo, blinding, and therapeutic misconception. We learned that these activities equipped $\mathrm{CAB}$ members to address questions that arose in the local community related to the importance of study procedures, such as how researchers could determine whether Truvada was effective for HIV prevention if the trial was providing risk-reduction counseling and condoms.

Outreach meetings and regular stakeholder forums within the larger community included education to increase understanding of HIV-prevention research. Although the mandate in GPP is to increase understanding of the specific trial and of biomedical HIV-prevention research in general, we also found it essential to lay a foundation of basic information about HIV - including how the virus is transmitted and existing ways of preventing HIV transmission - before providing any education about trials and research. We also found a need for broader, rather than only HIV-specific, education at some sites and that it was necessary to interweave trial-focused education with public forums on other relevant health issues.

In Bloemfontein, for example, outreach activities at hot spots where sex workers worked included referrals to health services, distribution of print materials on other sexually transmitted infections (STIs) and tuberculosis (TB), and distribution of male and female condoms. In Pretoria, community educators participated in the South African Health Calendar Days, such as World Tuberculosis Day, International Women's Day, and 16 Days of Activism against Gender Violence, as an opportunity to educate the larger community about FEM-PrEP, HIV-prevention research, and general health education on HIV, STIs, and
TB. The educators also networked closely with campaigns for HIV counseling and testing and other high-profile health campaigns to saturate the community with educational messages about research and FEM-PrEP.

\section{Communications plan}

GPP advises that communications planning should focus on "strategies that will increase broad awareness of the trial, facilitate dissemination and understanding of correct information about trial design, conduct, and results, and coordinate communication between the research team and relevant stakeholders." 6

The FEM-PrEP communications team was experienced in research networks, other multisite HIV-prevention trials, interfacing with the media and advocacy organizations, and participating in communities-of-practice groups, positioning it to effectively manage communications planning at local, national, and international levels, in conjunction with site teams. The role of community program staff in communications planning was locally focused and included identifying key local stakeholders to be contacted about any emerging news about FEM-PrEP and monitoring local issues and perceptions over the course of the trial (including locallanguage media). We found that having the sites' community program staff be part of a proactive communications team with experience at local and international levels facilitated communications planning and implementation at the local level. For example, all sites developed a site-specific standard operating procedure for the designated media spokesperson based on guidance and with oversight from the communications team.

FHI 360 staff and the sites community program staff also collaborated to modify a series of educational resources from the National Institutes of Health, the Global Campaign for Microbicides, and the International AIDS Vaccine Initiative in order to develop frequently-asked-question documents and fact sheets on clinical trials and PrEP trials, FEM-PrEP, and gender issues in PrEP research. These documents were used to train $\mathrm{CAB}$ members, FEM-PrEP staff, and local community stakeholders. Adapting them as needs emerged over the course of the trial further ensured their utility on the ground. Talking points were also developed to inform participants, local stakeholders, $\mathrm{CAB}$ members, and the community after results of microbicide and other PrEP trials were released. We also found that making use of written materials previously developed by reputable organizations in the field of HIV-prevention research was time- and cost-effective and ensured quality. For example, AVAC's fact sheet on data 
safety and monitoring boards proved invaluable in the basics of clinical trial trainings.

\section{Issues management plan}

GPP recommends that the issue management plan describe "how research teams intend to manage issues of concern or any unexpected developments that may emerge before, during, or after the trial, including those that could limit the support for, or success of, the specific trial or future biomedical HIV prevention trials." 6

Given lessons learned from the controversies related to the Cambodia and Cameroon PrEP trials and the potential for controversy regarding FEM-PrEP or any placebo-controlled biomedical HIV-prevention trial, issues management planning was addressed proactively as part of the overall FEM-PrEP communications plan. The plan outlined how the sites would respond rapidly and effectively to controversy and other issues as they arose. This included identifying, for example, anticipated controversial questions that might arise and how to answer them, how to recognize controversial issues as they emerged in the local community during the trial, communication activities to build consensus or support among opinion leaders and key stakeholders, and preparing site-specific communication to be led by community program staff and the $\mathrm{CABs} / \mathrm{CAG}$ regarding community concerns, rumors, and misinformation. A rapid-response contact grid was also developed that identified who should be contacted, by whom, and in what order in the event of controversies, issues, or rumors. $\mathrm{CAB} / \mathrm{CAG}$ members also played a significant role by reporting on emerging rumors in the community and their sources, and by relaying key messages and information back to the community. Upon learning of any rumors, community program site staff conducted outreach to address the rumors quickly before they were more widely circulated.

We found that being prepared for issues that had been anticipated - through the development of and training on the communication materials already described - allowed site community program staff to implement planned strategies effectively to address rumors when they emerged.

\section{Trial closure and results dissemination}

GPP guidelines specify that "[e]ffectively engaging relevant stakeholders about trial closure and results dissemination in a transparent process is essential for building trust and lays a positive foundation for future research. In the event that a trial is stopped early or unexpectedly, research team-initiated dialogue with relevant stakeholders will minimize the risk of misinformation." ${ }^{6}$
From the beginning of the trial, community program staff sought to manage expectations among local stakeholders in case the data safety and monitoring board found it necessary to stop the trial, whether for safety reasons, due to positive effectiveness results, or due to futility. All sites developed detailed a priori plans for results dissemination, including communication planning and message development. Following the April 2011 announcement of the early closure of FEM-PrEP and the March 2012 announcement of final results, all sites conducted comprehensive and systematic dissemination (Table 2). The order of stakeholder prioritization for dissemination consisted of notifying study participants first, followed by (in no particular order) local officials, $\mathrm{CABs} / \mathrm{CAG}$, ethics committees, regulatory bodies, public health professionals and the scientific community, and the local study community (including NGOs and community-based organizations).

We found that throughout the trial and upon dissemination of FEM-PrEP results, it was essential to contextualize FEM-PrEP in relationship to other ongoing biomedical HIVprevention research. This enabled local stakeholders to have a better understanding of what was happening in the field. We also wanted to help stakeholders know how to interpret the FEM-PrEP results in the face of potentially conflicting and confusing evidence of effectiveness from other trials. Community members' reactions to other trial results included confusion over their importance in relationship to FEM-PrEP, underscoring the need to explain the relevance of the outcomes of these studies and the contributions each would bring to HIV prevention.

\section{Discussion}

We sought to examine the FEM-PrEP community program activities in the context of the 2011 GPP guidelines and reflect on practical considerations for their implementation. All guidelines considered were implementable in the context of FEM-PrEP. A summary of our considerations for each guideline appears in Table 3.

In our reflection on the feasibility of implementing the guidelines, we identified two cross-cutting themes. First, we identified a need for stakeholder education to go beyond the 2011 GPP recommendation to increase research literacy in the specific biomedical HIV-prevention trial at hand and in clinical research in general. We found that local stakeholders required a base of information on HIV transmission and HIV prevention before any other education or engagement related to the trial could take place. This was both an ethical concern - given that some stakeholders were at higher risk of HIV infection and may have been in need of basic information on HIV prevention - and a practical one, given 
Table 3 Practical considerations for implementation of the GPP guidelines

I. Formal stakeholder advisory mechanisms in the form of CABs/CAG were feasible to set up at trial sites. However, maintaining these groups as a strong mechanism for facilitating dialogue with the research team required (I) community program staff that were experienced with stakeholder engagement at the local level, and (2) the participation of $C A B$ members in setting meeting agendas. Beyond the formal CAB/CAG mechanism, we found that informal stakeholder advisory mechanisms were especially effective for managing emerging issues in the local community, such as the need for greater engagement with political and community leaders and health personnel, and to disseminate messages designed specifically to address men's concerns. The documentation of these outreach meetings and two-way communication with the FHI 360 research team was essential for ensuring that consistent messages about the trial were communicated.

2. Regardless of the type of stakeholder engagement appropriate for each stakeholder, informing them about PrEP, PrEP research, and the FEM-PrEP trial through community education was always a baseline requirement.

3. Stakeholder education within both CABs/CAG and the local community had to combine information about the FEM-PrEP trial and HIV-prevention research with more general education about HIV and HIV prevention. This was especially the case when community program staff made use of existing forums and events to provide trial- and research-related education.

4. Development and implementation of the communication plan was facilitated by leadership at the sponsor level, with community program staff working in tandem with the communication team to plan and implement local activities. Adapting previously developed written informational materials was time- and cost-effective, and contributed to the high quality of the informational materials we used. These materials were also useful as dynamic documents, being further adapted as needs emerged.

5. Being prepared for potential issues that could pose problems at the site level - by anticipating problematic issues and preparing materials detailing how to respond - facilitated issues management and freed staff to be responsive to new rumors and problems as they emerged.

6. For trial closure and results dissemination, contextualizing FEM-PrEP in relationship to other ongoing biomedical HIV-prevention trials facilitated the community's understanding of FEM-PrEP. We also found that preparing local communities for the possibility of early trial closure throughout the trial was helpful in encouraging the community's understanding when early closure did occur.

Abbreviations: GPP, good participatory practice; CAB, community advisory board; CAG, community advisory group; FEM-PrEP, Preexposure Prophylaxis Trial for HIV Prevention among African Women; PrEP, preexposure prophylaxis.

that information about biomedical HIV-prevention research can only make sense in the context of a base of knowledge about HIV transmission and prevention. We also found that it made sense to incorporate education about HIV transmission and PrEP research in discussions of other health issues relevant to local stakeholders.

This need for a holistic approach to community stakeholder engagement that focuses on more than successful research implementation has been discussed by others. ${ }^{10,11}$ Community stakeholder engagement that utilizes existing community infrastructure such as HIV services has also been suggested, ${ }^{12}$ indicating a need to integrate trial-related information with other relevant education and services. Although some clinical researchers may feel that stakeholder education in broader topic areas falls outside the purview of stakeholder engagement for biomedical HIV-prevention research, we consider it to be a benefit consistent with the GPP guiding principle of integrity in which "maximizing benefits for community stakeholders" is advocated. We recommend that the next version of GPP address this issue of the need for broader HIV-related education.

The second cross-cutting point that emerged was the utility of doing as much anticipatory preparation as possible in the areas of communications planning, issues management, and trial closure and results dissemination. We attribute our ability to do this in FEM-PrEP to the integration of the community program with social and behavioral research, which in turn was integrated into the clinical research. More specifically, the site-preparedness social and behavioral research (implemented at three of the four sites) systematically identified local concerns that could have an impact on local stakeholder support for and understanding of the trial. These data, together with information collected among formal and informal stakeholder advisory mechanisms in the community program, informed the development of messages and strategies by researchers, the communication team, and community program staff. We believe that these messages contributed to the successful conduct of the trial at each site despite the early closure. This is an example of the integrated approach to prevention-science research recommended in other work, such as MacQueen and Cates (2005). ${ }^{13}$

Even though the primary analysis of clinical trial data revealed that adherence to the study product was too low to demonstrate the effectiveness of Truvada for HIV prevention among the study population, we do not believe that the community program failed. The community program was intended to create a supportive environment for women participating in the clinical trial, including for women who wanted to take the study drug, through community education about the purpose of the trial and by addressing the community's concerns about the trial. The FEM-PrEP researchers are currently exploring the reasons for low adherence among former FEM-PrEP participants, including any role that the community may have played.

FEM-PrEP was the first HIV-prevention trial to describe its community program in the context of specific GPP 
guidelines, but we join other HIV-prevention trials in responding to the GPP call for increased attention to local community stakeholder engagement. ${ }^{12,14-17}$ Although the 2007 GPP guidelines came out after the SBC protocol (of which the community program work was a part) had been approved and implementation had begun at some sites, the FEM-PrEP team was aware of their development and in support of efforts to establish them as normative through its portfolio of trial activities. Nonetheless, even though FEM-PrEP funders and researchers provided notable support of the community program, we believe that there is still progress to be made on the continuum towards a stakeholder engagement program that builds true partnerships with the local community, as outlined in Chung and Lounsbury (2006). ${ }^{18}$

Stakeholder engagement has become increasingly important in biomedical research for HIV prevention. The GPP guidelines provide an important and feasible framework for this work.

\section{Acknowledgments}

The authors are particularly grateful to the FEM-PrEP site $\mathrm{CABs} / \mathrm{CAG}$, study participants, and community partners and members who participated in the dialogue about FEM-PrEP over 5 years. In addition, we would like to thank the following people for their commitment to supporting and implementing comprehensive community programs at their sites: Jesse Asewe (Bondo), Theo Segoe and Ilse Reblin (Bloemfontein), Rachel Manongi and Temu Lucky (Arusha), and Ross Malamatsho and Joseph Skhosana (Pretoria). Finally, we would like to extend a special thank you to Kate MacQueen, Michael Szpir, Holly Burke, and Cindy Geary at FHI 360, for taking the time to read and provide feedback on our drafts. Thanks also to Donna McCarraher for her support of the authors.

\section{Disclosure}

The authors report no conflicts of interest in this work.

\section{References}

1. McGrory E, Irvin A, Heise L. Research Rashomon: Lessons Learned from the Cameroon Pre-exposure Prophylaxis Trial Site. Seattle: Program for Appropriate Technology in Health; 2009.
2. Singh JA, Mills EJ. The abandoned trials of pre-exposure prophylaxis for HIV: what went wrong? PLoS Med. 2005;2(9):e234.

3. Mills E, Rachlis B, Wu P, Wong E, Wilson K, Singh S. Media reporting of tenofovir trials in Cambodia and Cameroon. BMC Int Health Hum Rights. 2005;5:6.

4. Page-Shafer K, Saphonn V, Sun LP, Vun MC, Cooper DA, Kaldor JM. HIV prevention research in a resource-limited setting: the experience of planning a trial in Cambodia. Lancet. 2005;366(9495):1499-1503.

5. Mack N, Robinson ET, MacQueen KM, Moffett J, Johnson LM. The exploitation of "Exploitation" in the tenofovir prep trial in Cameroon: lessons learned from media coverage of an HIV prevention trial. J Empir Res Hum Res Ethics. 2010;5(2):3-19.

6. Joint United Nations Programme on HIV/AIDS. Good Participatory Practice: Guidelines for Biomedical HIV Prevention Trials. Geneva: UNAIDS; 2011.

7. Robinson E, Baron D, Heise L, Moffett J, Harlan S. Communications Handbook for Clinical Trials. Seattle: Program for Appropriate Technology in Health; 2010.

8. Van Damme L, Corneli A, Ahmed K, et al. Preexposure prophylaxis for HIV infection among African women. N Engl J Med. 2012;367(5): 411-422.

9. Rivera R, Borasky D, Carayon F, et al. Research Ethics Training Curriculum for Community Representatives. Durham (NC): Family Health International; 2004.

10. Minkler M, Blackwell AG, Thompson M, Tamir H. Community-based participatory research: implications for public health funding. $\mathrm{Am} J$ Public Health. 2003;93(8):1210-1213.

11. Minkler M. Ethical challenges for the "outside" researcher in community-based participatory research. Health Educ Behav. 2004;31(6):684-697.

12. Ellen JM, Wallace M, Sawe FK, Fisher K. Community engagement and investment in biomedical HIV prevention research for youth: rationale, challenges, and approaches. J Acquir Immune Defic Syndr. 2010;54 Suppl 1:S7-S11.

13. MacQueen KM, Cates $\mathrm{W}$ Jr. The multiple layers of prevention science research. Am J Prev Med. 2005;28(5):491-495.

14. Shagi C, Vallely A, Kasindi S, et al. A model for community representation and participation in HIV prevention trials among women who engage in transactional sex in Africa. AIDS Care. 2008;20(09): 1039-1049.

15. Morin SF, Maiorana A, Koester KA, Sheon NM, Richards TA. Community consultation in HIV prevention research: a study of community advisory boards at 6 research sites. J Acquir Immune Defic Syndr. 2003;33(4):513-520.

16. Strauss RP, Sengupta S, Quinn SC, et al. The role of community advisory boards: involving communities in the informed consent process. Am J Public Health. 2001;91(12):1938-1943.

17. White R, Chileshe M, Dawson L, et al. Fostering community understanding of sufficient benefit and early stopping for a phase 2B HIV prevention clinical trial in Africa. Clin Trials. 2011;8(1):103-111.

18. Chung K, Lounsbury DW. The role of power, process, and relationships in participatory research for statewide HIV/AIDS programming. Soc Sci Med. 2006;63(8):2129-2140.
Open Access Journal of Clinical Trials

\section{Publish your work in this journal}

The Open Access Journal of Clinical Trials is an international, peerreviewed, open access journal publishing original research, reports, editorials, reviews and commentaries on all aspects of clinical tria design, management, legal, ethical and regulatory issues, case record form design, data collection, quality assurance and data auditing

\section{Dovepress}

methodologies. The manuscript management system is completely online and includes a very quick and fair peer-review system, which is all easy to use. Visit http://www.dovepress.com/testimonials.php to read real quotes from published authors. 